Hargrove, D. S., (2007) Keeping a rural psychology presence alive. Clinical Psychology: Science \& Practice, 14(3): 304-305 (Sep 2007). Published by Wiley-Blackwell (ISSN: 0969-5893). [The definitive version is available at www3.interscience.wiley.com]

\title{
Keeping a Rural Psychology Presence Alive
}

David S. Hargrove

\begin{abstract}
Rural communities were brought into the national mental health picture in the 1960s in President John F. Kennedy's Community Mental Health Centers Act of 1962. The delivery of services and the human resource application of that act designated rural areas as underserved, in need of some attention. Training programs in psychology, psychiatry, social work, and nursing focused some efforts to produce professional people who could work in the rural environment. As federal initiatives in community mental health have changed, the stimuli for the continued development of rural services have lagged. A thread of concern has lingered, however, as the article by Jameson and Blank (2007) demonstrates.
\end{abstract}


A thin, sinewy string of clinicians, researchers, and administrators wax and wane in the belief that rural people and communities deserve the elusive care for mental disorders. For 30 years calls for changes in the configurations of human resources, rethinking of professional ethical provisions, clarity of the implications of definitional controversies, and expanding the scopes of practice of several professions have been made to influence mental health service delivery in rural environments (Hargrove, 1982; Hargrove \& Breazeale, 1993; Schwartz, 2004; United States Department of Agriculture, 2007) The rural task panel of the President's Commission on Mental Health (1978) inspired some self-examination in medicine and psychiatry, psychology, nursing, and social work, the four core professions of mental health designated by the National Institute of Mental Health. A spurt of programmatic and research activity concerning rural mental health care resulted from this attention. Jameson and Blank (2007) demonstrate important continuity and new explanations with those early people who were concerned about rural people and environments.

The suggestion that psychology may provide a catalyst in the development of mental health care in rural areas turns a bright light on the potential for addressing some of the lingering problems. Psychology is both an academic discipline and a profession with the potential for service to the rural environment because, at its core, it rests on empirical science. Virtually all psychological practitioners are trained in a model that brings science and practice into some informative relationship with each other. In the best case, practice and science are in dialogue. Psychology's reliance on sound methodology permeates its various subdisciplines, like clinical, counseling, industrial-organizational, and educational psychology. The discipline of psychology is focused on the development and testing of theory, leading to application to specific populations and settings.

Jameson and Blank provide a perspective of the issues the administration and delivery of mental health care in rural areas have encountered. Furthermore, they brought current issues of the development of psychology as a profession to light in relationship to rural service delivery. Specifically, they discuss the potential implications of the development of empirically supported treatments for mental disorders and the drive toward medication prescriptive authority, both under heated discussion in organized psychology, to bear on rural mental health service delivery. They clearly documented both the historical base and the changing scene of professional psychology, particularly as it relates to rural matters. 
Although both the discipline and profession of psychology are in flux, rural communities are also changing. Two of the most obvious changes to rural environments are in the demographics of rural America (United States Department of Agriculture, 2007). First, an increasing number of people are choosing to live in some rural communities, moving from urban centers, seeking different lifestyles (John, 1993). As progressive technology renders location less important for employment, more urban and suburban families are moving to rural communities. Consequences of this trend are changes in the demographic configuration of previously insular communities, shifts in the power structure and local political organizations, and increased demands on community infrastructures. Second, because of increased immigration, the number of ethnic minorities in rural communities has risen sharply ( John, 1993). This results in issues of assimilation of different cultures into those previously insular communities.

Important questions arise from these changes. At the broader community level, what is the impact of these demographic changes on the quality of life as perceived by the residents? What is the impact on the governmental infrastructure that influences the provision of health, mental health, social, and educational services to the population? As diversity increases, are there differential responses to the modes of service delivery? Are assumptions about the nature and consequences of mental illness that underlie traditional service delivery systems consistent with those systems that must serve diverse communities? Are traditional assessment and intervention strategies for diverse groups relevant? Or are they tied to assumptions about a population that no longer is relevant? These and other questions arise for policymakers, administrators, researchers, and clinicians who wish to serve rural people in rural communities. They are not the same questions that rural investigators have studied in the past, but they are consistent with the spirit of seeking systems and methods that are effective in reaching and serving rural people.

The discipline and profession of psychology is well positioned to ask and help answer these questions. The lingering question that comes from the past and emerges into the present is what institutions are going to allow those questions and answers to be sought. Over the years, the federal government, state governments, and local authorities have shifted responsibility for the relevance of rural care to the point that it is no longer clear who accepts that responsibility. In our system of government, 
it seems that all are. Of course, that answer points out the political nature of the questions and the answers. The questions of rural mental health began in politics in the 1960s when Will Edgerton and Bill Hollister (Hollister, Edgerton, \& Hunter, 1985) developed the North Carolina articles, and they continue into the twenty-first century when Michael Blank et al., among others, seek solutions to age-old problems.

\section{REFERENCES}

Jameson, J. P., \& Blank, M. B. (2007). The role of clinical psychology in rural mental health services: Defining problems and developing solutions. Clinical Psychology: Science and Practice,14 , 283-298.

Hargrove, D. S. (1982). The rural psychologist as generalist: A challenge for professional identity. Professional Psychology: Research and Practice, 13 , 302-308.

Hargrove, D. S., \& Breazeale, R. L. (1993). Psychologists and rural services: Addressing a new agenda. Professional Psychology: Research and Practice, 24 (3), 319-324.

Hollister, W. G., Edgerton, J. W., \& Hunter, R. H. (1985).

Alternative services in community mental health: Programs and Processes . Chapel Hill: University of North Carolina Press.

John, P. L. C. (1993). Population migration in rural America, QB 93-95. Beltsville, MD: Rural Information Center.

Schwartz, T. J. (2004). Convergence between psychology and public health in a rural community. Journal of Rural Community Psychology, E7 (2).

Task Panel on Rural Mental Health. (1978). In President's Commission on Mental Health, report to the president (vol. 3). Washington, DC: United States Government Printing Office.

United States Department of Agriculture. (2007). Measuring rurality: What is rural? Briefing Rooms . http:// www.ers.usda.gov/Briefing/Rurality/WhatisRural/ 